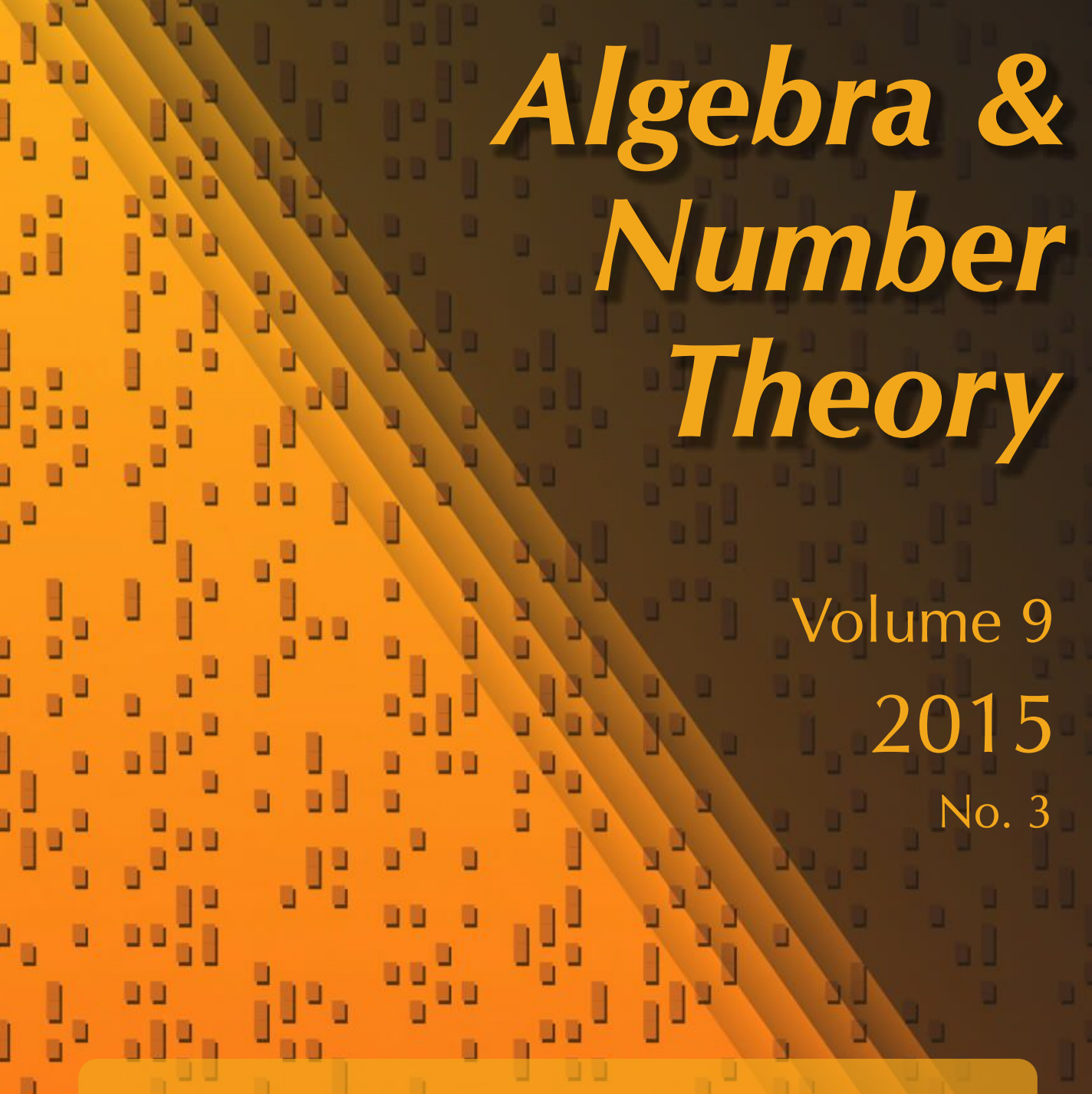

\title{
The torsion group of endotrivial modules
}

Jon F. Carlson and Jacques Thévenaz.

」

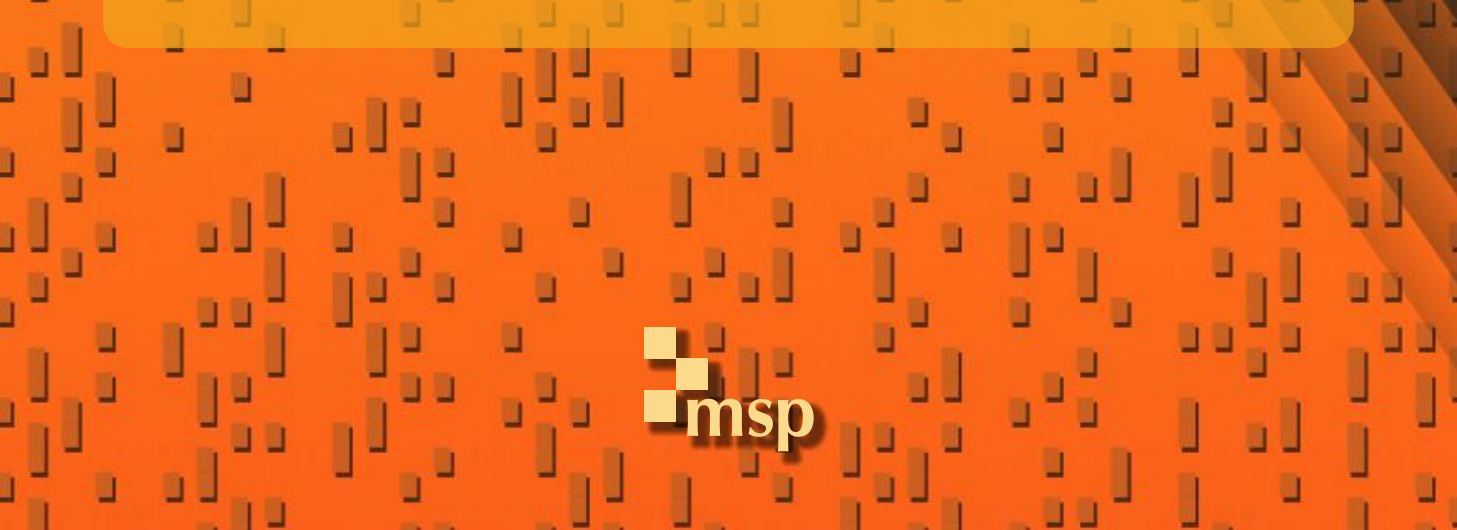




\title{
The torsion group of endotrivial modules
}

\author{
Jon F. Carlson and Jacques Thévenaz
}

\begin{abstract}
Let $G$ be a finite group and let $T(G)$ be the abelian group of equivalence classes of endotrivial $k G$-modules, where $k$ is an algebraically closed field of characteristic $p$. We determine, in terms of the structure of $G$, the kernel of the restriction map from $T(G)$ to $T(S)$, where $S$ is a Sylow $p$-subgroup of $G$, in the case when $S$ is abelian. This provides a classification of all torsion endotrivial $k G$-modules in that case.
\end{abstract}

\section{Introduction}

Endotrivial modules for a finite group $G$ over a field $k$ of prime characteristic $p$ play a significant role in modular representation theory. Among other things, they form an important part of the Picard group of self-equivalences of the stable category $\operatorname{stmod}(k G)$ of finitely generated $k G$-modules modulo projectives. They are modules which have universal deformation rings [de Smit and Lenstra 1997]. The endotrivial modules have been classified in the case that $G$ is a $p$-group [Carlson and Thévenaz $2004 ; 2005]$ and various results have appeared since for some specific families of groups [Carlson et al. 2006; 2009; 2010; 2011; 2013; 2014a; 2014b; Mazza 2007; Mazza and Thévenaz 2007; Navarro and Robinson 2012; Lassueur and Mazza 2015]. Recently, another line of research has developed that is concerned with the classification of all endotrivial modules which are simple [Robinson 2011; Lassueur et al. 2013; Lassueur and Malle 2015].

The main purpose of this paper is to classify all torsion endotrivial modules when a Sylow $p$-subgroup is abelian. We let $G$ be a finite group and $T(G)$ be the abelian group of equivalence classes of endotrivial $k G$-modules, where $k$ is an algebraically closed field of characteristic $p$. Let $S$ be a Sylow $p$-subgroup of $G$. We fix the notation

$$
K(G)=\operatorname{Ker}\left\{\operatorname{Res}_{S}^{G}: T(G) \longrightarrow T(S)\right\} .
$$

One of the main open questions is to describe this kernel explicitly, and we achieve this goal here in the case that $S$ is abelian. Actually, $K(G)$ is known to be equal to

Carlson is partially supported by a grant from NSF.

MSC2010: 20C20.

Keywords: modular representation theory. 
the torsion subgroup of $T(G)$ in most cases. Specifically, this happens whenever $S$ is not cyclic, generalized quaternion, or semidihedral - because, if we exclude these three cases, then $T(S)$ is torsion-free by [Carlson and Thévenaz 2005]. The excluded cases are treated in [Mazza and Thévenaz 2007; Kawata 1993; Carlson et al. 2013].

Let $N=N_{G}(S)$ denote the normalizer of a Sylow $p$-subgroup $S$ of $G$. It is known that the restriction map $\operatorname{Res}_{N}^{G}: K(G) \rightarrow K(N)$ is injective, induced by the Green correspondence, and the problem is to describe its image. In fact, $K(N)$ consists of all one-dimensional representations of $N$ and the main difficulty is to know which of them lie in the image of $\operatorname{Res}_{N}^{G}$; that is, which of them have Green correspondents that are endotrivial. Indeed, if $J$ is the intersection of the kernels of the one-dimensional $k N$-modules whose Green correspondents are endotrivial, then $K(G)$ is isomorphic to the dual group $(N / J)^{*}$. So the problem of finding $K(G)$ comes down to the question of what is $J$.

Another approach was introduced by Balmer [2013], in which he shows that $K(G)$ is isomorphic to the group $A(G)$ of all weak homomorphisms $G \rightarrow k^{\times}$ (defined below in Section 3). Balmer's method was used effectively in [Carlson et al. 2014a] to compute $K(G)$ in some crucial cases for $G$ a special linear group. The method involved the construction of a system of local subgroups $\left\{\rho^{i}(Q)\right\}$ indexed on the collection of nontrivial subgroups $Q$ of the Sylow subgroup $S$ of $G$ and for $i \geq 1$ (see Section 4 for the definition). These subgroups are in the kernels of all weak homomorphisms and we show here that $\rho^{i}(S) \subseteq N_{G}(S)$ is in the kernel of all one-dimensional representations of $N_{G}(S)$ whose Green correspondents are endotrivial modules. That is, $\rho^{i}(S) \subseteq J$, for $J$ defined as above.

Hence, the question of determining $K(G)$ becomes: is $J$ equal to $\rho^{\infty}(S)$, the limit of the system? The answer is yes in all examples that we know. The main theorem of this paper says that the answer is yes whenever the Sylow $p$-subgroup of $G$ is abelian. Indeed, we prove more. We show that $J=\rho^{2}(S)$, the subgroup of $N=N_{G}(S)$ generated by the commutator subgroup [ $\left.N, N\right], S$, and all intersections $N \cap\left[N_{G}(Q), N_{G}(Q)\right]$ for $Q$ a nontrivial subgroup of $S$. Thus, $K(G) \cong\left(N / \rho^{2}(S)\right)^{*}$ in the case that $S$ is abelian. Balmer's characterization of $K(G)$ in terms of weak homomorphisms is crucial for the proof, which appears in Section 5.

In Section 6 we show that the main theorem can be used to describe $K(G)$ explicitly when $S$ is cyclic. An extension of the main theorem to the case where the normalizer $N$ of $S$ controls $p$-fusion is given in Section 7. The paper ends with some examples of simple or almost simple groups where the subgroup $K(G)$ is not trivial.

\section{Endotrivial modules and the restriction to the Sylow subgroup}

Throughout this paper, $k$ denotes an algebraically closed field of prime characteristic $p$ and $G$ is a finite group. We assume that all modules are finitely generated. If 
$M$ and $L$ are $k G$-modules, the notation $M \cong L \oplus$ (proj) means that $M$ is isomorphic to the direct sum of $L$ with some projective $k G$-module, which might be zero. We write $k$ for the trivial $k G$-module. Unless otherwise specified, the symbol $\otimes$ is the tensor product $\otimes_{k}$ of the underlying vector spaces. In the case that $M$ and $L$ are $k G$-modules, the tensor product $M \otimes L$ is a $k G$-module with $G$ acting by the diagonal action on the factors.

We assume that $G$ has order divisible by $p$ and we let $S$ be a Sylow $p$-subgroup of $G$. Recall that a $k G$-module $M$ is endotrivial provided its endomorphism al$\operatorname{gebra} \operatorname{End}_{k}(M)$ is isomorphic (as a $k G$-module) to the direct sum of the trivial module $k$ and a projective $k G$-module. In other words, $M$ is endotrivial if and only if $\operatorname{Hom}_{k}(M, M) \cong M^{*} \otimes M \cong k \oplus$ (proj), where $M^{*}$ denotes the $k$-dual of $M$. Any endotrivial module $M$ splits as the direct sum $M=M_{0} \oplus$ (proj) for an indecomposable endotrivial $k G$-module $M_{0}$, which is unique up to isomorphism. We let $T(G)$ be the set of equivalence classes of endotrivial $k G$-modules for the equivalence relation

$$
M \sim L \Longleftrightarrow M_{0} \cong L_{0} .
$$

Every equivalence class contains a unique indecomposable module up to isomorphism. The tensor product induces an abelian group structure on the set $T(G)$, written additively as $[M]+[L]=[M \otimes L]$. The zero element of $T(G)$ is the class $[k]$ of the trivial module, while the inverse of the class of a module $M$ is the class of the dual module $M^{*}$. The group $T(G)$ is known to be a finitely generated abelian group.

Lemma 2.1 [Carlson et al. 2011, Lemma 2.3]. Let $K(G)$ be the kernel of the restriction map $\operatorname{Res}_{S}^{G}: T(G) \longrightarrow T(S)$ to a Sylow p-subgroup $S$.

(a) $K(G)$ is a finite subgroup of $T(G)$.

(b) $K(G)$ is the entire torsion subgroup $T T(G)$ of $T(G)$, provided $S$ is not cyclic, generalized quaternion, or semidihedral.

We say that a $k G$-module $M$ has trivial Sylow restriction if the restriction of $M$ to a Sylow $p$-subgroup $S$ has the form $M \downarrow_{S}^{G} \cong k \oplus$ (proj). Any such module is endotrivial and is the direct sum of an indecomposable trivial source module and a projective module. Thus $M$ has trivial Sylow restriction if and only if its class [ $M$ ] belongs to $K(G)$.

Proposition 2.2. Suppose that a finite group $H$ has a nontrivial normal p-subgroup. Then every indecomposable $\mathrm{kH}$-module with trivial Sylow restriction has dimension one.

Proof. The proof is a straightforward application of the Mackey formula. The details appear in Lemma 2.6 in [Mazza and Thévenaz 2007]. 
In the situation of Proposition 2.2, for any one-dimensional $k H$-module $L$ we write $\chi_{L}: H \rightarrow k^{\times}$for the corresponding group homomorphism (representation).

Our next result is an easy application of the Green correspondence. For details, see Proposition 2.6 in [Carlson et al. 2006].

Proposition 2.3. Let $S$ be a Sylow p-subgroup of $G$ and let $N=N_{G}(S)$.

(a) The restriction map $\operatorname{Res}_{N}^{G}: T(G) \rightarrow T(N)$ is injective, induced by the Green correspondence.

(b) In particular, the restriction map $\operatorname{Res}_{N}^{G}: K(G) \rightarrow K(N)$ is injective.

We emphasize that, if $M$ and $L$ are $k G$-modules with trivial Sylow restriction then, by Proposition 2.2, $M \downarrow_{N}^{G} \cong U \oplus$ (proj) and $L \downarrow_{N}^{G} \cong V \oplus$ (proj), where $U$ and $V$ are $k N$-modules of dimension one. Here $U$ is the Green correspondent of $M$, $V$ is the Green correspondent of $L$, and we see automatically that $U \otimes V$ is the Green correspondent of $(M \otimes L)_{0}$, the unique indecomposable nonprojective direct summand of $M \otimes L$.

We know that $K\left(N_{G}(S)\right)$ consists exactly of all one-dimensional representations of $N$. The main problem is to know which of them are in the image of the restriction map from $K(G)$. In other words, given a one-dimensional $k N$-module $U$, we need to know when its Green correspondent $M$ is endotrivial.

Another way of viewing the situation is the following:

Proposition 2.4. Let $S$ denote a Sylow p-subgroup of $G$ and let $N=N_{G}(S)$. Let $J \subseteq N$ be the intersection of the kernels of all one-dimensional $k N$-modules $U$ such that the Green correspondent $M$ of $U$ is an endotrivial $k G$-module. That is, $J$ is the intersection of the kernels of $U$ such that $[U]$ is in the image of the restriction $\operatorname{Res}_{N}^{G}: T(G) \rightarrow T(N)$. Then $K(G) \cong(N / J)^{*} \cong \operatorname{Hom}\left(N / J, k^{\times}\right)$.

Proof. The restriction map $\operatorname{Res}_{N}^{G}: K(G) \rightarrow K(N)$, being injective, gives an isomorphism between $K(G)$ and a subgroup $A \subseteq K(N) \cong \operatorname{Hom}\left(N, k^{\times}\right)$, the group of one-dimensional representations of $N$. But $A$ is isomorphic to the dual group of $N / J$, where $J$ is the intersection of the kernels of the elements of $A$.

From the proposition, we see that the problem of characterizing the group $K(G)$ is equivalent to finding the group $J$. The main purpose of this paper is to offer a possible candidate for the subgroup $J$. In addition, we prove that the candidate is, in fact, equal to $J$ in the case that the Sylow $p$-subgroup $S$ is abelian.

\section{Weak homomorphisms and the kernel of restriction}

Balmer [2013] provided a new characterization of the group $K(G)$ in terms of the group of weak $S$-homomorphisms, which we describe in this section. Note that Balmer's construction is more general than the one that we use here. He defined 
"weak $H$-homomorphisms" for any subgroup $H$ containing $S$. Because, we only deal with the case that $H=S$ in this paper, we call them "weak homomorphisms". Balmer [2015] has expanded his results, but here we only need the formulation in [Balmer 2013].

For notation, recall that ${ }^{g} S$ denotes the conjugate subgroup $g S g^{-1}$, while $S^{g}$ denotes $g^{-1} S g$.

Definition 3.1. A map $\chi: G \rightarrow k^{\times}$is called a weak homomorphism ("weak $S$ homomorphism" in the language of [Balmer 2013]) if it satisfies the following three conditions:

(a) If $s \in S$, then $\chi(s)=1$.

(b) If $g \in G$ and $S \cap{ }^{g} S=\{1\}$, then $\chi(g)=1$.

(c) If $a, b \in G$ and if $S \cap{ }^{a} S \cap{ }^{a b} S \neq\{1\}$, then $\chi(a b)=\chi(a) \chi(b)$.

The product of two weak homomorphisms $\chi$ and $\psi$ is defined pointwise by $(\chi \psi)(g)=\chi(g) \psi(g)$ and is again a weak homomorphism. The set $A(G)$ of all weak homomorphisms is an abelian group under this operation.

Theorem 3.2 [Balmer 2013]. The groups $K(G)$ and $A(G)$ are isomorphic.

The isomorphism is explicit and is described in detail in [Balmer 2013]. In particular, it is shown that, given a weak homomorphism $\chi$, there is a certain norm-type formula using $\chi$ that constructs a homomorphism from the permutation module $k(G / S)$ to itself, whose image is the endotrivial module associated to $\chi$.

It is important, in what follows, to understand Balmer's isomorphism on restriction to a subgroup $H$ having a nontrivial normal $p$-subgroup. This is our next result. For notation, given a finite group $H$, we let $\diamond(H)$ be the smallest normal subgroup of $H$ such that $H / \diamond(H)$ is an abelian $p^{\prime}$-group. In other words $\diamond(H)=[H, H] S$ is the subgroup of $H$ generated by the commutator subgroup $[H, H]$ and by a Sylow $p$-subgroup $S$ of $H$.

Proposition 3.3. Suppose that a finite group $H$ has a nontrivial normal $p$-subgroup.

(a) Every weak homomorphism $\chi: H \rightarrow k^{\times}$is a group homomorphism.

(b) The isomorphism $K(H) \rightarrow A(H)$ maps the class of a one-dimensional $k H$ module $M$ to the corresponding group representation $H \rightarrow k^{\times}$.

(c) The group $A(H)$ is isomorphic to the group of one-dimensional representations of $H$, that is, the dual group $(H / \diamond(H))^{*}$.

Proof. To prove (a), let $Q$ be a nontrivial normal $p$-subgroup of $H$. For any $a, b \in G$, the subgroup $S \cap{ }^{a} S \cap{ }^{a b} S$ is nontrivial because it contains $Q={ }^{a} Q={ }^{a b} Q$. Thus the third condition in Definition 3.1 implies that $\chi(a b)=\chi(a) \chi(b)$.

Statement (b) follows from the construction of the isomorphism given in Section 2.5 of [Balmer 2013]. See also Corollary 5.1 in [Balmer 2013]. The proof 
of (c) is straightforward, because the image of any group homomorphism $H \rightarrow k^{\times}$ is contained in the subgroup of $k^{\times}$consisting of roots of unity, namely the group of all $p^{\prime}$-roots of unity since $k$ is algebraically closed and has characteristic $p$.

Proposition 3.4. Let $N=N_{G}(S)$, where $S$ is the Sylow p-subgroup of $G$. The restriction map $\operatorname{Res}_{N}^{G}: A(G) \rightarrow A(N)$ is injective.

Proof. This follows from Proposition 2.3, Balmer's isomorphism in Theorem 3.2, and the fact that this isomorphism is natural.

\section{A system of local subgroups}

In this section, we discuss the properties of a sequence of subgroups $\rho^{i}(Q) \subseteq N_{G}(Q)$, where $Q$ is a nontrivial $p$-subgroup of $S$ and $i \geq 1$. The construction of the sequence was first presented in [Carlson et al. 2014a], though the version here is slightly different. The definition of the subgroups $\rho^{i}(Q)$ depends not only on $N_{G}(Q)$, but involves all normalizers of nontrivial subgroups of the Sylow subgroup $S$ of $G$.

The definition proceeds inductively as follows. We fix a Sylow $p$-subgroup $S$ of $G$. For any nontrivial subgroup $Q$ of $S$, let

$$
\rho^{1}(Q):=\diamond\left(N_{G}(Q)\right) .
$$

As before, $\diamond\left(N_{G}(Q)\right)$ is the product of the commutator subgroup of $N_{G}(Q)$ and a Sylow $p$-subgroup of $N_{G}(Q)$. The original version in [Carlson et al. 2014a] uses simply the commutator subgroup of $N_{G}(Q)$. For $i>1$, we let

$$
\rho^{i}(Q):=\left\langle N_{G}(Q) \cap \rho^{i-1}\left(Q^{\prime}\right) \mid\{1\} \neq Q^{\prime} \subseteq S\right\rangle,
$$

the subgroup generated by the subgroups $N_{G}(Q) \cap \rho^{i-1}\left(Q^{\prime}\right)$ for all nontrivial subgroups $Q^{\prime}$ of $S$. This contains $\rho^{i-1}(Q)$, so we have a nested sequence of subgroups

$$
\rho^{1}(Q) \subseteq \rho^{2}(Q) \subseteq \rho^{3}(Q) \subseteq \cdots \subseteq N_{G}(Q) .
$$

Since $G$ is finite, the sequence eventually stabilizes, and we let $\rho^{\infty}(Q)$ be the limit subgroup of the sequence $\left\{\rho^{i}(Q) \mid i \geq 1\right\}$, namely their union.

The definition of the subgroups $\rho^{i}(Q)$ was originally motivated in [Carlson et al. 2014a] by the following observation:

Proposition 4.1. Suppose that $\chi: G \rightarrow k^{\times}$is a weak homomorphism as defined in the last section. If $x \in \rho^{i}(Q)$ for some $i \geq 1$ and for some nontrivial subgroup $Q$ of $S$, then $\chi(x)=1$.

Proof. In the case that $i=1$, the statement is a trivial consequence of the definition of a weak homomorphism. That is, $\chi(x)=1$ for any $x \in Q$ by Definition 3.1(a), and is a homomorphism to an abelian group when restricted to $N_{G}(Q)$ by Definition 3.1(c). So assume that $i>1$ and that $\chi(x)=1$ for all $x \in \rho^{i-1}(Q)$ for all nontrivial 
subgroups $Q \subseteq S$. Then $\chi(x)=1$ for all $x \in N_{G}(Q) \cap \rho^{i-1}\left(Q^{\prime}\right)$ for any nontrivial subgroups $Q$ and $Q^{\prime}$. Thus, $\chi(x)=1$ for all $x \in \rho^{i}(Q)$, and the proposition is proved by induction.

Suppose that $M$ is a $k G$-module with trivial Sylow restriction. By Proposition 2.2, for any nontrivial subgroup $Q$ of $S$ there is a one-dimensional $k N_{G}(Q)$-module $L_{Q}$ such that $M \downarrow_{N_{G}(Q)}^{G} \cong L_{Q} \oplus$ (proj). We write $\chi_{Q}=\chi_{L_{Q}}$ for the corresponding group homomorphism $\chi_{Q}: N_{G}(Q) \rightarrow k^{\times}$.

The next result encapsulates the main idea of this section. It should be compared with Proposition 4.1.

Proposition 4.2. Suppose that $M$ is a $k G$-module with trivial Sylow restriction, let $Q$ be a nontrivial subgroup of $S$ and let $\chi_{Q}: N_{G}(Q) \rightarrow k^{\times}$be defined as above. Then $\rho^{\infty}(Q)$ is contained in the kernel of $\chi_{Q}$.

Proof. We prove, by induction on $i$, that if $x \in \rho^{i}(Q)$ then $\chi_{Q}(x)=1$. In the case that $i=1$, the result is a consequence of the fact that $\rho^{1}(Q)=\diamond\left(N_{G}(Q)\right)$ is in the kernel of every one-dimensional character on $N_{G}(Q)$. Inductively, we assume that the lemma is true for $\rho^{j}\left(Q^{\prime}\right)$ whenever $j<i$ and $1 \neq Q^{\prime} \subseteq S$. Because $x \in \rho^{i}(Q)$, we have that $x=x_{1} \cdots x_{m}$ for some $m$, where $x_{t} \in N_{G}(Q) \cap \rho^{i-1}\left(Q_{t}\right)$, for some nontrivial subgroup $Q_{t}$ of $S$, for each $t=1, \ldots, m$. For each $t$ we consider the restriction of $M$ to $N_{G}(Q) \cap N_{G}\left(Q_{t}\right)$. Using the notation above, this yields

$$
M \downarrow_{N_{G}(Q) \cap N_{G}\left(Q_{t}\right)}^{G} \cong L_{Q} \downarrow_{N_{G}(Q) \cap N_{G}\left(Q_{t}\right)}^{N_{G}(Q)} \oplus \text { (proj) } \cong L_{Q_{t}} \downarrow_{N_{G}(Q) \cap N_{G}\left(Q_{t}\right)}^{N_{G}\left(Q_{t}\right)} \oplus \text { (proj). }
$$

The intersection $N_{G}(Q) \cap N_{G}\left(Q_{t}\right)$ contains the center of $S$, which is a nontrivial $p$-subgroup. Thus, any indecomposable projective module over $N_{G}(Q) \cap N_{G}\left(Q_{t}\right)$ has dimension divisible by $p$. Therefore, by the Krull-Schmidt theorem,

$$
L_{Q} \downarrow_{N_{G}(Q) \cap N_{G}\left(Q_{t}\right)}^{N_{G}(Q)} \cong L_{Q_{t}} \downarrow_{N_{G}(Q) \cap N_{G}\left(Q_{t}\right)}^{N_{G}\left(Q_{t}\right)} .
$$

For all $t$, it follows by induction that $\chi_{Q}\left(x_{t}\right)=\chi_{Q_{t}}\left(x_{t}\right)=1$, because $x_{t} \in \rho^{i-1}\left(Q_{t}\right)$. Thus $\chi_{Q}(x)=1$, as asserted.

The main theorem of this section is a special case of the above.

Theorem 4.3. Suppose that $M$ is an endotrivial $k G$-module with trivial Sylow restriction, i.e., $M \downarrow_{S}^{G} \cong k \oplus$ (proj). Then $M \downarrow_{\rho^{\infty}(S)}^{G} \cong k \oplus$ (proj).

There are several immediate corollaries.

Corollary 4.4. Suppose that $\rho^{\infty}(S)=N_{G}(S)$. Then the only indecomposable $k G$-module with trivial Sylow restriction is the trivial module; that is, $K(G)=\{0\}$.

Proof. If $M$ is a $k G$-module with trivial Sylow restriction, then our assumption implies that $M \downarrow_{N_{G}(S)}^{G} \cong k \oplus$ (proj). Because $M$ is indecomposable, it is the Green correspondent of the trivial module $k_{N_{G}(S)}$. Thus $M$ is the trivial module. 
Corollary 4.5. Suppose that $Q$ is a normal subgroup of $S$ and that, for some $i$, $N_{G}(S) \subseteq \rho^{i}(Q)$. Then the only indecomposable $k G$-module with trivial Sylow restriction is the trivial module. In other words, $K(G)=\{0\}$.

Proof. Under the hypothesis, we have that $N_{G}(S) \subseteq \rho^{i+1}(S)$, and we are done by the previous corollary.

Note in the above corollary that $Q$ being normal in $S$ does not assure that $N_{G}(S)$ is a subgroup of $N_{G}(Q)$. However, if $Q$ is characteristic in $S$, then this is a certainty.

Corollary 4.6. Suppose that $Q$ is a characteristic subgroup of $S$ and that, for some $i, N_{G}(Q)=\rho^{i}(Q)$. Then the only indecomposable $k G$-module with trivial Sylow restriction is the trivial module. In other words, $K(G)=\{0\}$.

\section{Abelian Sylow subgroup}

The purpose of this section is to prove our main theorem, which describes $K(G)$ when a Sylow $p$-subgroup $S$ of $G$ is abelian. Specifically, we show the following:

Theorem 5.1. Suppose that a Sylow p-subgroup $S$ of $G$ is abelian. Let $N=N_{G}(S)$.

(a) The image of the restriction map $\operatorname{Res}_{N}^{G}: A(G) \rightarrow A(N)$ consists exactly of all group homomorphisms $N_{G}(S) \rightarrow k^{\times}$having $\rho^{2}(S)$ in their kernel.

(b) $K(G) \cong A(G) \cong\left(N_{G}(S) / \rho^{2}(S)\right)^{*}$.

(c) $\rho^{2}(S)=\rho^{\infty}(S)$.

In other words, the theorem says that if the Sylow subgroup $S$ of $G$ is abelian, then the subgroup $J$ of Proposition 2.4 is equal to $\rho^{2}(S)$ and that $K(G) \cong\left(N / \rho^{2}(S)\right)^{*}$.

Note that, by Proposition 4.1, the restriction to $N_{G}(Q)$ of any weak homomorphism $\phi: G \rightarrow k^{\times}$is a homomorphism having $\rho^{2}(Q)$ in its kernel. Our task then is to prove a very strong converse, namely, that any group homomorphism $\chi: N=N_{G}(S) \rightarrow k^{\times}$having $\rho^{2}(S)$ in its kernel is the restriction to $N$ of a weak homomorphism.

We need a couple of preliminary results before beginning the proof. The first observation is essential to our efforts.

Lemma 5.2. Let $S$ be a Sylow p-subgroup of a finite group $H$.

(a) The inclusion map $N_{H}(S) \rightarrow H$ induces an isomorphism

$$
N_{H}(S) / N_{H}(S) \cap \diamond(H) \cong H / \diamond(H) .
$$

(b) For any group homomorphism $\phi: N_{H}(S) \rightarrow k^{\times}$having $N_{H}(S) \cap \diamond(H)$ in its kernel, there exists a unique group homomorphism $\psi: H \rightarrow k^{\times}$whose restriction to $N_{H}(S)$ is equal to $\phi$. 
Proof. By the definition of $\diamond(H)$, the Sylow $p$-subgroup $S$ is contained in $\diamond(H)$. The Frattini argument yields $N_{H}(S) \diamond(H)=H$, proving (a). Then (b) follows from (a).

Lemma 5.3 (Burnside's fusion theorem). Let $S$ be an abelian Sylow p-subgroup of $G$ and suppose that $R$ is a nontrivial subgroup of $S \cap{ }^{g} S$.

(a) There exists $c \in C_{G}(R)$ and $n \in N_{G}(S)$ such that $g=c n$.

(b) If $g=c^{\prime} n^{\prime}$ with $c^{\prime} \in C_{G}(R)$ and $n^{\prime} \in N_{G}(S)$, then there exists $d \in N_{G}(S) \cap C_{G}(R)$ such that $c^{\prime}=c d$ and $n^{\prime}=d^{-1} n$.

Proof. Statement (a) is essentially Burnside's theorem. For the proof, observe that $S$ and ${ }^{g} S$ are Sylow $p$-subgroups of $C_{G}(R)$, hence conjugate by an element $c \in C_{G}(R)$. Then $n=c^{-1} g$ normalizes $S$. Statement (b) follows by defining $d=c^{-1} c^{\prime}=n n^{-1}$.

The essence of the proof of Theorem 5.1 is the following:

Proposition 5.4. Suppose that a Sylow p-subgroup $S$ of $G$ is abelian, and let $N=N_{G}(S)$. Let $\chi: N \rightarrow k^{\times}$be a homomorphism whose kernel contains $\rho^{2}(S)$. Then there is a unique weak homomorphism $\theta: G \rightarrow k^{\times}$whose restriction to $N$ is equal to $\chi$.

Proof. Let $Q$ be a nontrivial subgroup of $S$, and let $H=C_{G}(Q)$, the centralizer of $Q$ in $G$. Since $S$ is abelian, $S \subseteq H \subseteq N_{G}(Q)$. Clearly, $\diamond(H)=[H, H] S \subseteq \diamond\left(N_{G}(Q)\right)$, hence $\chi$ vanishes on $N_{H}(S) \cap \diamond(H) \subseteq \rho^{2}(S)$ by assumption. By Lemma 5.2, there exists a unique group homomorphism

$$
\psi_{Q}: H=C_{G}(Q) \longrightarrow k^{\times}
$$

that coincides with $\chi$ on the subgroup $N_{H}(S)=N_{G}(S) \cap C_{G}(Q)=N \cap H$.

We define $\theta: G \rightarrow k^{\times}$by the following rule. First, we set $\theta(g)=1$ if $S \cap{ }^{g} S=1$. If $S \cap{ }^{g} S \neq\{1\}$, we use Lemma 5.3 and write $g=c n$, with $c \in C_{G}\left(S \cap{ }^{g} S\right)$ and $n \in N_{G}(S)$. Then let

$$
\theta(g)=\psi_{S \cap g S}(c) \chi(n) .
$$

In order to prove that $\theta$ is well defined, we must consider another decomposition $g=c^{\prime} n^{\prime}$, with $c^{\prime} \in C_{G}\left(S \cap{ }^{g} S\right)$ and $n^{\prime} \in N_{G}(S)$, and show that the algorithm produces the same result for $\theta(g)$. In fact, we prove more: that the algorithm produces the same result even if we replace $S \cap{ }^{g} S$ by a proper nontrivial subgroup. That is, assume that $R$ is any nontrivial subgroup of $S \cap{ }^{g} S$ and write $g=c^{\prime} n^{\prime}$, with $c^{\prime} \in C_{G}(R)$ and $n^{\prime} \in N_{G}(S)$. We claim that

$$
\psi_{S \cap \cap^{g} S}(c) \chi(n)=\psi_{R}\left(c^{\prime}\right) \chi\left(n^{\prime}\right) .
$$


In order to prove the claim, we observe that $C_{G}\left(S \cap{ }^{g} S\right) \subseteq C_{G}(R)$ because $R \subseteq S \cap{ }^{g} S$. So we have two decompositions $g=c n=c^{\prime} n^{\prime}$ with $c, c^{\prime} \in C_{G}(R)$ and $n, n^{\prime} \in N_{G}(S)$. By Lemma 5.3, there exists $d \in N_{G}(S) \cap C_{G}(R)$ such that $c^{\prime}=c d$ and $n^{\prime}=d^{-1} n$. Thus,

$$
\psi_{R}\left(c^{\prime}\right) \chi\left(n^{\prime}\right)=\psi_{R}(c d) \chi\left(d^{-1} n\right)=\psi_{R}(c) \psi_{R}(d) \chi(d)^{-1} \chi(n)=\psi_{R}(c) \chi(n)
$$

because $d \in N_{G}(S) \cap C_{G}(R)$ and we know that $\psi_{R}$ and $\chi$ coincide on $N_{G}(S) \cap C_{G}(R)$. We next observe that the uniqueness of $\psi_{S \cap{ }^{g} S}: C_{G}\left(S \cap{ }^{g} S\right) \rightarrow k^{\times}$implies that it must be equal to the restriction to $C_{G}\left(S \cap{ }^{g} S\right)$ of the group homomorphism $\psi_{R}: C_{G}(R) \rightarrow k^{\times}$. Therefore $\psi_{R}(c)=\psi_{S \cap \cap_{S}}(c)$, completing the proof of the claim.

Our next task is to prove that $\theta$ is a weak homomorphism. If $s \in S$, then $S \cap S=S$ and we have $C_{G}(S) \subseteq N_{G}(S)$. We use the decomposition $s=1 \cdot s$ with $1 \in C_{G}(S)$ and $s \in N_{G}(S)$ and we get $\theta(s)=\chi(s)$. But $\chi$ vanishes on $S$ because $S \subseteq \diamond(N)$. Therefore $\theta(s)=1$, proving the first condition for a weak homomorphism. The second condition is obvious, since $\theta(g)=1$ if $S \cap{ }^{g} S=1$, by definition.

Assume that $a, b \in G$ with $S \cap \cap^{a} S \cap^{a b} S \neq\{1\}$. We must show that $\theta(a b)=\theta(a) \theta(b)$. Let $R=S \cap{ }^{a} S \cap{ }^{a b} S$, and note that $R \subseteq S \cap{ }^{a} S$. Using what we have proved, we can write

$$
\theta(a)=\psi_{R}(c) \chi(n), \quad \text { where } a=c n, c \in C_{G}(R), n \in N_{G}(S) .
$$

Next notice that $R \subseteq{ }^{a} S \cap{ }^{a b} S$, so that $R^{a} \subseteq S \cap{ }^{b} S$. We write

$$
\theta(b)=\psi_{R^{a}}(d) \chi(m), \quad \text { where } b=d m, d \in C_{G}\left(R^{a}\right), m \in N_{G}(S) .
$$

It follows that ${ }^{a} b={ }^{a} d^{a} m$, with ${ }^{a} d \in C_{G}(R),{ }^{a} m \in N_{G}\left({ }^{a} S\right)$. Therefore

$$
a b={ }^{a} b a={ }^{a} d^{a} m a={ }^{a} d c c^{-1} a m={ }^{a} d c n m,
$$

because $c^{-1} a=n$. But ${ }^{a} d c \in C_{G}(R)$, because $d \in C_{G}\left(R^{a}\right)$ and $n m \in N_{G}(S)$. So we obtain

$$
\theta(a b)=\psi_{R}\left({ }^{a} d c\right) \chi(n m)=\psi_{R}\left({ }^{a} d\right) \psi_{R}(c) \chi(n) \chi(m)=\psi_{R}(c) \chi(n) \psi_{R}\left({ }^{a} d\right) \chi(m) .
$$

Now we claim that $\psi_{R}\left({ }^{a} d\right)=\psi_{R^{a}}(d)$. Writing $a=c n$, we first find that

$$
\psi_{R}\left({ }^{a} d\right)=\psi_{R}\left(c^{n} d c^{-1}\right)=\psi_{R}(c) \psi_{R}\left({ }^{n} d\right) \psi_{R}\left(c^{-1}\right)=\psi_{R}\left({ }^{n} d\right),
$$

because $k^{\times}$is commutative. Moreover, writing $\operatorname{conj}_{n}(x)=n x n^{-1}$, we observe that the composite

$$
C_{G}\left(R^{a}\right)=C_{G}\left(R^{n}\right) \stackrel{\text { onj }_{n}}{\longrightarrow} C_{G}(R) \stackrel{\psi_{R}}{\longrightarrow} k^{\times}
$$


has a restriction to $N_{G}(S) \cap C_{G}\left(R^{n}\right)$ equal to

$$
N_{G}(S) \cap C_{G}\left(R^{n}\right) \stackrel{\text { onj }_{n}}{\longrightarrow} N_{G}(S) \cap C_{G}(R) \stackrel{\chi}{\longrightarrow} k^{\times} .
$$

Hence, it is equal to the map

$$
N_{G}(S) \cap C_{G}\left(R^{n}\right) \stackrel{\chi}{\longrightarrow} k^{\times},
$$

because $n \in N_{G}(S)$ and $\chi\left(n \times n^{-1}\right)=\chi(n) \chi(x) \chi\left(n^{-1}\right)=\chi(x)$ by commutativity of $k^{\times}$. It follows that $\psi_{R} \circ \operatorname{conj}_{n}: C_{G}\left(R^{n}\right) \rightarrow k^{\times}$is the unique extension of $\chi: N_{G}(S) \cap C_{G}\left(R^{n}\right) \rightarrow k^{\times}$, hence equal to the homomorphism $\psi_{R^{n}}: C_{G}\left(R^{n}\right) \rightarrow k^{\times}$. In other words, $\psi_{R}\left({ }^{n} d\right)=\psi_{R}\left(\operatorname{conj}_{n}(d)\right)=\psi_{R^{n}}(d)$. Finally,

$$
\psi_{R}\left({ }^{a} d\right)=\psi_{R}\left({ }^{n} d\right)=\psi_{R^{n}}(d)=\psi_{R^{a}}(d),
$$

as claimed.

Returning to the computation of $\theta(a b)$, we find

$$
\theta(a b)=\psi_{R}(c) \chi(n) \psi_{R}\left({ }^{a} d\right) \chi(m)=\psi_{R}(c) \chi(n) \psi_{R^{a}}(d) \chi(m)=\theta(a) \theta(b) .
$$

This completes the proof of the third condition for a weak homomorphism. Thus $\theta: G \rightarrow k^{\times}$is a weak homomorphism, and we have proved the proposition.

Proof of Theorem 5.1. To prove (a), we recall that any weak homomorphism on $N$ is a homomorphism. The image under the restriction map $\operatorname{Res}_{N}^{G}: A(G) \rightarrow A(N)$ of any element of $A(G)$ must be a homomorphism with $\rho^{2}(S)$ in its kernel. The previous proposition says that the restriction map must be injective and surjective onto this subset.

Statement (b) follows immediately from (a) and the injectivity of the restriction map (Proposition 3.4). The proof of (c) — that is, the equality $\rho^{2}(S)=\rho^{\infty}(S)-$ follows from (a) and the fact that $\rho^{\infty}(S)$ is in the kernel of any weak homomorphism, by Theorem 4.3 .

All of the experimental evidence suggests that something like Theorem 5.1 should be true in general. Hence, we suggest the following question:

Question 5.5. Suppose that $G$ is any finite group with Sylow p-subgroup $S$. Let $N=N_{G}(S)$, and let $J \subseteq N$ be the intersection of the kernels of all one-dimensional $k N$-modules $U$ such that the Green correspondent of $U$ is an endotrivial $k G$-module. Is $J=\rho^{\infty}(S)$ ?

While it might be possible to prove an affirmative answer by means similar to those in the proof of Theorem 5.1, we should point out that at least two difficulties arise. The first is that the fusion theorem of Burnside does not hold in greater generality. It would have to be replaced with something like Alperin's fusion theorem, whose conditions are more complicated. In addition, as we see in Section 7, 
even a stringent assumption such as control of fusion by the normalizer of the Sylow subgroup $S$ does not readily lead to a generalization. Other assumptions seem to be necessary for the proof.

A second difficulty generalizing Theorem 5.1 is that the equality $\rho^{2}(S)=\rho^{\infty}(S)$ does not hold in general. An example is $G_{2}(5)$ for $k$ a field of characteristic 3. Computer calculations using Magma [Bosma and Cannon 1996] show that $\rho^{3}(S)=\rho^{\infty}(S)=N_{G}(S)$, but $\rho^{2}(S)$ is a proper subgroup of $N_{G}(S)$.

\section{The cyclic case}

If a Sylow $p$-subgroup $S$ of $G$ is cyclic, then the structure of $K(G)$ is determined in Theorem 3.6 of [Mazza and Thévenaz 2007]. In this section, we show that this result can be recovered using Theorem 5.1. We prove the following:

Theorem 6.1. Suppose that a Sylow p-subgroup $S$ of $G$ is cyclic. Let $Z$ be the unique subgroup of $S$ of order $p$. Then $K(G) \cong K\left(N_{G}(Z)\right) \cong\left(N_{G}(Z) / \diamond\left(N_{G}(Z)\right)\right)^{*}$. Proof. For any subgroup $Q$ such that $Z \subseteq Q \subseteq S$, we have that $N_{G}(Q) \subseteq N_{G}(Z)$. Hence $\diamond\left(N_{G}(Q)\right) \subseteq \diamond\left(N_{G}(Z)\right)$, and

$$
\rho^{2}(S)=N_{G}(S) \cap \diamond\left(N_{G}(Z)\right) .
$$

By Lemma 5.2 applied to the subgroup $H=N_{G}(Z)$, we have an isomorphism

$$
N_{G}(S) / \rho^{2}(S)=N_{G}(S) / N_{G}(S) \cap \diamond\left(N_{G}(Z)\right) \cong N_{G}(Z) / \diamond\left(N_{G}(Z)\right) .
$$

Since $K(G) \cong\left(N_{G}(Z) / \rho^{2}(S)\right)^{*}$ by Theorem 5.1 , we obtain

$$
K(G) \cong\left(N_{G}(Z) / \diamond\left(N_{G}(Z)\right)\right)^{*},
$$

as required. This is also isomorphic to $K\left(N_{G}(Z)\right)$, by Proposition 3.3.

The isomorphism $K(G) \cong K\left(N_{G}(Z)\right)$ actually follows directly from the isomorphism $T(G) \cong T\left(N_{G}(Z)\right)$, which is a consequence of the fact that $N_{G}(Z)$ is strongly $p$-embedded in $G$ (see Lemma 3.5 in [Mazza and Thévenaz 2007]).

\section{Control of fusion}

Assume that the normalizer $N_{G}(S)$ of a Sylow $p$-subgroup $S$ of $G$ controls $p$-fusion. One may wonder if Theorem 5.1 still holds under this assumption. The analysis of the proof shows that we need more, as follows.

Theorem 7.1. Suppose that the normalizer $N=N_{G}(S)$ of a Sylow p-subgroup $S$ of $G$ controls $p$-fusion. Assume, in addition, that, for any subgroup $Q$ of $S$, we have $N_{H}(S) \diamond(H)=H$, where $H=C_{G}(Q)$ and $N_{H}(S)=N_{G}(S) \cap H$. Then the following hold: 
(a) The image of the restriction map $\operatorname{Res}_{N_{G}(S)}^{G}: A(G) \rightarrow A\left(N_{G}(S)\right)$ consists exactly of all group homomorphisms $N_{G}(S) \rightarrow k^{\times}$having $\rho^{2}(S)$ in their kernel.

(b) $K(G) \cong A(G) \cong\left(N_{G}(S) / \rho^{2}(S)\right)^{*}$.

(c) $\rho^{2}(S)=\rho^{\infty}(S)$.

Proof. The proof is exactly the same as that of Theorem 5.1, with the following observations. The assumption on each group $H=C_{G}(Q)$ implies that the conclusions of Lemma 5.2 hold. Thus the use of Lemma 5.2 remains valid. More precisely, for any group homomorphism $\chi: N_{G}(S) \rightarrow k^{\times}$vanishing on $N_{H}(S) \cap \diamond(H)$, there exists a unique group homomorphism $\psi_{Q}: C_{G}(S) \rightarrow k^{\times}$which coincides with $\chi$ on the subgroup $N_{H}(S)=N_{G}(S) \cap H$. Here $S$ is not necessarily contained in $H$ (while it is when $S$ is abelian), so the Frattini argument cannot be applied as it was in Lemma 5.2. However, our assumption allows us to make the argument work.

On the other hand, the assumption on control of fusion means exactly that the conclusions of Lemma 5.3 hold. Thus, the use of Lemma 5.3 remains valid, and the whole proof goes through.

\section{Examples}

If $H$ is a strongly $p$-embedded subgroup of $G$, then any one-dimensional representation of $H$ has a Green correspondent which is endotrivial (see Proposition 2.8 and Remark 2.9 in [Carlson et al. 2006]). This fact was used to produce torsion endotrivial modules of dimension greater than one in various cases, in particular for groups of Lie type of rank one in the defining characteristic (Proposition 5.2 in [Carlson et al. 2006]) and for groups with a cyclic Sylow p-subgroup (Lemma 3.5 in [Mazza and Thévenaz 2007]).

However, there are other cases when torsion endotrivial modules of dimension greater than one occur. Several examples are given in [Lassueur and Mazza 2015] for various sporadic groups. The purpose of this section is to provide two explicit examples for classical groups with an abelian Sylow $p$-subgroup. We first start by an easy case.

Example 8.1 $(\operatorname{PSL}(2, q)$ in characteristic 2$)$. Let $G=\operatorname{PSL}(2, q)$ in characteristic 2 and assume that $q \equiv 3$ or 5 modulo 8, so that a Sylow 2-subgroup $S$ of $G$ is a Klein four-group. Then $C_{G}(S)=S$ has index 3 in $N_{G}(S)$, hence $\diamond\left(N_{G}(S)\right)=S$. Any subgroup $C$ of order 2 satisfies $N_{G}(C)=C_{G}(C)$ and

$$
S \subseteq N_{G}(S) \cap \diamond\left(N_{G}(C)\right) \subseteq N_{G}(S) \cap N_{G}(C)=N_{G}(S) \cap C_{G}(C)=S .
$$

Therefore $N_{G}(S) \cap \diamond\left(N_{G}(C)\right)=S$, and it follows that $\rho^{2}(S)=\rho^{1}(S)=S$. By Theorem 5.1, $K(G)$ is the dual group of $N_{G}(S) / S$, which is cyclic of order 3 . Hence $T T(G)=K(G) \cong \mathbb{Z} / 3 \mathbb{Z}$. 
For our second example, we compute the torsion part of the group of endotrivial modules over the group $G=\operatorname{PSL}(3, q)$ in characteristic 3 , in the case that $q \equiv 4$ or 7 modulo 9. In this case, the Sylow 3-subgroup of $G$ is elementary abelian of order 9. The point is to show that the torsion subgroup $T T(G)=K(G)$ of the group of endotrivial module is isomorphic to $(\mathbb{Z} / 2 \mathbb{Z})^{2}$, which has three nontrivial elements.

For notation, we use an overline to indicate the class in $G$ of an element in $H=\operatorname{SL}(3, q)$. Let $\zeta$ denote a cubed root of unity in $\mathbb{F}_{q}$. We fix the following elements of $H$ :

$$
a=\left[\begin{array}{lll}
1 & 0 & 0 \\
0 & \zeta & 0 \\
0 & 0 & \zeta^{2}
\end{array}\right], \quad x=\left[\begin{array}{lll}
0 & 1 & 0 \\
0 & 0 & 1 \\
1 & 0 & 0
\end{array}\right], \quad u=\left[\begin{array}{ccc}
1 & 1 & 1 \\
1 & \zeta & \zeta^{2} \\
1 & \zeta^{2} & \zeta
\end{array}\right], \quad v=\left[\begin{array}{ccc}
\zeta & 1 & 1 \\
\zeta & \zeta & \zeta^{2} \\
1 & \zeta & 1
\end{array}\right] .
$$

Then it is not difficult to verify the following:

Lemma 8.2. The subgroup $\langle a, x\rangle$ is a Sylow 3-subgroup of $H=\operatorname{SL}(3, q)$, and hence the Sylow 3-subgroup of $G$ is $S=\langle\bar{a}, \bar{x}\rangle$. In addition, we have the relations:

(1) $u^{-1} x u=a$,

(2) $u^{-1} a u=x^{-1}$,

(3) $v^{-1} x v=a^{2} v$,

(4) $v^{-1} a v=\zeta(a x)^{-1}$.

In particular, the elements $\bar{u}$ and $\bar{v}$ are in the normalizer of $S$, and each acts on $S$ by exchanging the four maximal subgroups in pairs. The commutator $\sigma=u^{-1} v^{-1} u v$ acts on $S$ by inverting $\bar{a}$ and $\bar{x}$, and hence also inverts every nonidentity element.

Now we consider the centralizers and normalizers. Let $z \in H$ be the scalar matrix with nonzero entries equal to $\zeta$. Then $\langle z\rangle$ is the kernel of the natural homomorphism of $H$ onto $G$. A general principle here is that, if $y$ is an element of $H$ such that $\bar{y}$ commutes with $\bar{x}$, then, for some $j, a^{j} y$ is in the centralizer of $x$, and the same holds with $a$ and $x$ exchanged.

It is easy to see that the centralizer of $a$ in $H$ is the Levi subgroup $L$ of all diagonal matrices of determinant one. Then the normalizer of $\langle a, z\rangle$ is the normalizer of $L$ which is generated by $L, x$ and the element $\sigma$, the commutator of $u$ and $v$. The centralizer of $x$, which has the same order as that of $a$, consists of all elements of the form

$$
\left[\begin{array}{lll}
c & d & e \\
e & c & d \\
d & e & c
\end{array}\right]
$$

having determinant one (that is, $c^{3}+d^{3}+e^{3}-3 c d e=1$ ). Then, the normalizer of $\langle x, z\rangle$ is generated by this centralizer, $a$ and $\sigma$. 
Thus we can prove the following:

Proposition 8.3. The Sylow 3-subgroup $S$ is self-centralizing. Its normalizer is generated by $\bar{a}, \bar{x}, \bar{u}, \bar{v}$ and $\bar{\sigma}$.

Proof. We can see that the centralizer of $S$ is generated by the classes $\bar{a}, \bar{x}$ and the classes of the intersection of the centralizers of $a$ and $x$ in $H$. However, this intersection consists only of the elements of $\langle z\rangle$. The elements $\bar{u}$ and $\bar{v}$ permute the maximal subgroups of $S$ transitively. So suppose that $\bar{y}$ is an element of the normalizer of $S$ and $y$ a preimage of $\bar{y}$ in $H$. By replacing $y$ by its product with a power of $u$ and/or a power of $v$ we may assume that $\bar{y}$ normalizes $\langle\bar{a}\rangle$. By replacing $\bar{y}$ by its product with $\sigma$, if necessary, we may assume that $\bar{y}$ centralizes $\bar{a}$. Multiplying $y$ by a power of $x$, if necessary, we may assume that $y$ has the form

$$
\left[\begin{array}{lll}
r & 0 & 0 \\
0 & s & 0 \\
0 & 0 & t
\end{array}\right]
$$

where $r s t=1$. Thus we have that

$$
y x y^{-1}=\left[\begin{array}{ccc}
0 & r^{2} t & 0 \\
0 & 0 & r s^{2} \\
s t^{2} & 0 & 0
\end{array}\right] .
$$

The point of this is that $\bar{y} \bar{x} \bar{y}^{-1}$ must be one of the elements $\bar{x}, \bar{a} \bar{x}$ or $\bar{a}^{2} \bar{x}$ and cannot be $\bar{x}^{2}, \bar{a} \bar{x}^{2}$ or $\bar{a}^{2} \bar{x}^{2}$. Because $y$ centralizes $a$, conjugation by $\bar{y}$ is an automorphism of order either one or three on $S$. Order 3 is not possible. Consequently, $\bar{y} \bar{x} \bar{y}^{-1}=\bar{x}$ and $\bar{y}$ centralizes $S$. This proves the proposition.

Now we are ready for the main theorem.

Theorem 8.4. Assume that $G=\operatorname{PSL}(3, q)$, where $q$ is congruent to 4 or 7 modulo 9 .

(a) Let $S$ be a Sylow 3-subgroup of $G$. Then $\rho^{\infty}(S)=\rho^{1}(S)=\left[N_{G}(S), N_{G}(S)\right]$.

(b) $T T(G)=K(G) \cong(\mathbb{Z} / 2 \mathbb{Z})^{2}$.

Proof. (a) First, $\rho^{1}(S)=\diamond\left(N_{G}(S)\right)=\left[N_{G}(S), N_{G}(S)\right]$ by definition and the fact that $\left[N_{G}(S), N_{G}(S)\right]$ has index prime to 3 in $N_{G}(S)$ (namely index 4). We also have that, for any subgroup $U$ of order 3 in $S, N_{G}(U) \cap N_{G}(S) \subseteq\left[N_{G}(S), N_{G}(S)\right]$. Consequently, again from the definition, we have that $\rho^{n}(S)=\left[N_{G}(S), N_{G}(S)\right]$ for all $n$.

(b) Since a Sylow 3-subgroup is abelian of rank two, $T T(G)=K(G)$ is isomorphic to the dual group of $N_{G}(S) / \rho^{2}(S)$ by Theorem 5.1. By (a), $\rho^{2}(S)=\left[N_{G}(S), N_{G}(S)\right]$ and, by Proposition 8.3, $N_{G}(S) /\left[N_{G}(S), N_{G}(S)\right] \cong\left(C_{2}\right)^{2}$, a Klein four-group, generated by the classes of $\bar{u}$ and $\bar{v}$. Its dual group (in additive notation) is isomorphic to $(\mathbb{Z} / 2 \mathbb{Z})^{2}$. 
Remark 8.5. In the case that $q=4$, the normalizer of the Sylow subgroup $S$ of $G$ is strongly 3 -embedded, and the result of the theorem could be deduced from that fact. In all other cases, $N_{G}(S)$ is not strongly 3 -embedded and it is not strongly 3 -embedded in any subgroup of $G$ that properly contains it.

Finally, it is not difficult to perform the computations of the subgroups $\rho^{i}(Q)$ for all subgroups $Q$ of the Sylow subgroup $S$ of $G$ on a computer using a standard computer algebra system. From this computation, the structure of $K(G)=T T(G)$ can in many cases be deduced using Theorem 5.1 or something similar. Below are a few calculations using Magma [Bosma and Cannon 1996]. In most cases, only a few seconds of computing time was required. The computing time depends on such things as the size of the permutation representation of $G$ and the number of subgroups of $S$. Here we list only groups where $K(G)$ is not trivial. The results should be compared with those of [Lassueur et al. 2013; Lassueur and Malle 2015; Lassueur and Mazza 2015]. The notation for the groups is the Atlas notation.

\begin{tabular}{ccc}
\hline $\mathrm{TT}(\mathrm{G})$ & Group & Characteristic $p$ \\
\hline \multirow{Z}{*}{$/ 2 \mathbb{Z}$} & $M_{23}, \mathrm{Ru}$ & 3 \\
$\mathbb{Z} / 2 \mathbb{Z} \times \mathbb{Z} / 2 \mathbb{Z}$ & $J_{2}, \mathrm{Suz}, 2 \mathrm{Suz}, 6 \mathrm{Suz}, \mathrm{Fi}_{22}, \mathrm{Fi}_{23}$ & 5 \\
& $M_{11}, M_{22}, M_{23}, \mathrm{HS}$ & 3 \\
$\mathbb{Z} / 4 \mathbb{Z}$ & $2 \mathrm{Ru}$ & 3 \\
$\mathbb{Z} / 2 \mathbb{Z} \times \mathbb{Z} / 4 \mathbb{Z}$ & $\mathrm{Co}_{3}, \mathrm{Sz}_{32}$ & 5 \\
$\mathbb{Z} / 8 \mathbb{Z}$ & $2 M_{22}, 4 M_{22}$ & 3 \\
$\mathbb{Z} / 24 \mathbb{Z}$ & $\mathrm{McL}$ & 5 \\
\hline
\end{tabular}

\section{Acknowledgment}

The authors are grateful to Paul Balmer and Caroline Lassueur for several useful discussions.

\section{References}

[Balmer 2013] P. Balmer, "Modular representations of finite groups with trivial restriction to Sylow subgroups", J. Eur. Math. Soc. (JEMS) 15:6 (2013), 2061-2079. MR 3120735 Zbl 1291.20009

[Balmer 2015] P. Balmer, "Stacks of group representations", J. Eur. Math. Soc. (JEMS) 17:1 (2015), 189-228. MR 3312406

[Bosma and Cannon 1996] W. Bosma and J. Cannon, "Handbook on Magma functions", University of Sydney, 1996, http://magma.maths.usyd.edu.au/magma/handbook/.

[Carlson and Thévenaz 2004] J. F. Carlson and J. Thévenaz, "The classification of endo-trivial modules”, Invent. Math. 158:2 (2004), 389-411. MR 2005e:20013 Zbl 1110.20004 
[Carlson and Thévenaz 2005] J. F. Carlson and J. Thévenaz, "The classification of torsion endo-trivial modules", Ann. of Math. (2) 162:2 (2005), 823-883. MR 2006f:20012 Zbl 1110.20005

[Carlson et al. 2006] J. F. Carlson, N. Mazza, and D. K. Nakano, "Endotrivial modules for finite groups of Lie type”, J. Reine Angew. Math. 595 (2006), 93-119. MR 2007c:20023 Zbl 1106.20007

[Carlson et al. 2009] J. F. Carlson, N. Mazza, and D. K. Nakano, "Endotrivial modules for the symmetric and alternating groups", Proc. Edinb. Math. Soc. (2) 52:1 (2009), 45-66. MR 2009k:20023 Zbl 1165.20005

[Carlson et al. 2010] J. F. Carlson, D. J. Hemmer, and N. Mazza, "The group of endotrivial modules for the symmetric and alternating groups”, Proc. Edinb. Math. Soc. (2) 53:1 (2010), 83-95. MR 2011d:20017 Zbl 1217.20004

[Carlson et al. 2011] J. F. Carlson, N. Mazza, and J. Thévenaz, "Endotrivial modules for $p$-solvable groups", Trans. Amer. Math. Soc. 363:9 (2011), 4979-4996. MR 2012f:20026 Zbl 1250.20007

[Carlson et al. 2013] J. F. Carlson, N. Mazza, and J. Thévenaz, "Endotrivial modules over groups with quaternion or semi-dihedral Sylow 2-subgroup", J. Eur. Math. Soc. (JEMS) 15:1 (2013), 157-177. MR 2998832 Zbl 1276.20007

[Carlson et al. 2014a] J. F. Carlson, N. Mazza, and D. K. Nakano, "Endotrivial modules for the general linear group in a nondefining characteristic”, Math. Z. 278:3-4 (2014), 901-925. MR 3278897 Zbl 06384008

[Carlson et al. 2014b] J. F. Carlson, N. Mazza, and J. Thévenaz, "Torsion-free endotrivial modules", J. Algebra 398 (2014), 413-433. MR 3123775 Zbl 06334760

[Kawata 1993] S. Kawata, "On Auslander-Reiten components for certain group modules", Osaka J. Math. 30:2 (1993), 137-157. MR 95c:20008 Zbl 0819.20007

[Lassueur and Malle 2015] C. Lassueur and G. Malle, "Simple endo-trivial modules for the linear, unitary and exceptional groups", preprint, 2015. arXiv 1501.00400

[Lassueur and Mazza 2015] C. Lassueur and N. Mazza, "Endotrivial modules for the sporadic simple groups and their covers", J. Pure Appl. Algebra (online publication February 2015).

[Lassueur et al. 2013] C. Lassueur, G. Malle, and E. Schulte, "Simple endotrivial modules for quasi-simple groups", J. Reine Angew. Math. (online publication November 2013).

[Mazza 2007] N. Mazza, "The group of endotrivial modules in the normal case", J. Pure Appl. Algebra 209:2 (2007), 311-323. MR 2007j:20010 Zbl 1117.20009

[Mazza and Thévenaz 2007] N. Mazza and J. Thévenaz, "Endotrivial modules in the cyclic case", Arch. Math. (Basel) 89:6 (2007), 497-503. MR 2009b:20015 Zbl 1155.20009

[Navarro and Robinson 2012] G. Navarro and G. R. Robinson, "On endo-trivial modules for $p$ solvable groups", Math. Z. 270:3-4 (2012), 983-987. MR 2892933 Zbl 1242.20004

[Robinson 2011] G. R. Robinson, "On simple endotrivial modules", Bull. Lond. Math. Soc. 43:4 (2011), 712-716. MR 2012g:20022 Zbl 1234.20003

[de Smit and Lenstra 1997] B. de Smit and H. W. Lenstra, Jr., "Explicit construction of universal deformation rings", pp. 313-326 in Modular forms and Fermat's last theorem (Boston, MA, 1995), edited by G. Cornell et al., Springer, New York, 1997. MR 1638482 Zbl 0907.13010

Communicated by David Benson

Received 2014-11-05 Revised 2015-01-28 Accepted 2015-02-27

jfc@math.uga.edu

Department of Mathematics, University of Georgia, Athens, GA 30602, United States

jacques.thevenaz@epfl.ch Section de mathématiques, EPFL, Station 8, $\mathrm{CH}-1015$ Lausanne, Switzerland 


\section{Algebra \& Number Theory}

msp.org/ant

\section{EDITORS}

MANAGING EDITOR

Bjorn Poonen

Massachusetts Institute of Technology

Cambridge, USA

\author{
EDITORIAL BOARD CHAIR \\ David Eisenbud \\ University of California \\ Berkeley, USA
}

\section{BOARD OF EDITORS}

Georgia Benkart

Dave Benson

Richard E. Borcherds

John H. Coates

J-L. Colliot-Thélène

Brian D. Conrad

Hélène Esnault

Hubert Flenner

Edward Frenkel

Andrew Granville

Joseph Gubeladze

Roger Heath-Brown

Craig Huneke

János Kollár

Yuri Manin

Barry Mazur

Philippe Michel
University of Wisconsin, Madison, USA

University of Aberdeen, Scotland

University of California, Berkeley, USA

University of Cambridge, UK

CNRS, Université Paris-Sud, France

University of Michigan, USA

Freie Universität Berlin, Germany

Ruhr-Universität, Germany

University of California, Berkeley, USA

Université de Montréal, Canada

San Francisco State University, USA

Oxford University, UK

University of Virginia, USA

Princeton University, USA

Northwestern University, USA

Harvard University, USA

École Polytechnique Fédérale de Lausanne
Susan Montgomery

Shigefumi Mori

Raman Parimala

Jonathan Pila

Anand Pillay

Victor Reiner

Peter Sarnak

Joseph H. Silverman

Michael Singer

Vasudevan Srinivas

J. Toby Stafford

Ravi Vakil

Michel van den Bergh

Marie-France Vignéras

Kei-Ichi Watanabe

Efim Zelmanov

Shou-Wu Zhang
University of Southern California, USA

RIMS, Kyoto University, Japan

Emory University, USA

University of Oxford, UK

University of Notre Dame, USA

University of Minnesota, USA

Princeton University, USA

Brown University, USA

North Carolina State University, USA

Tata Inst. of Fund. Research, India

University of Michigan, USA

Stanford University, USA

Hasselt University, Belgium

Université Paris VII, France

Nihon University, Japan

University of California, San Diego, USA

Princeton University, USA

\section{PRODUCTION}

production@msp.org

Silvio Levy, Scientific Editor

See inside back cover or msp.org/ant for submission instructions.

The subscription price for 2015 is US $\$ 255 /$ year for the electronic version, and $\$ 440 /$ year ( $+\$ 55$, if shipping outside the US) for print and electronic. Subscriptions, requests for back issues and changes of subscribers address should be sent to MSP.

Algebra \& Number Theory (ISSN 1944-7833 electronic, 1937-0652 printed) at Mathematical Sciences Publishers, 798 Evans Hall \#3840, c/o University of California, Berkeley, CA 94720-3840 is published continuously online. Periodical rate postage paid at Berkeley, CA 94704, and additional mailing offices.

ANT peer review and production are managed by EditFLOW ${ }^{\circledR}$ from MSP.

\section{PUBLISHED BY}

- mathematical sciences publishers

nonprofit scientific publishing

http://msp.org/

(C) 2015 Mathematical Sciences Publishers 


\section{Algebra \& Number Theory}

Volume $9 \quad$ No. $3 \quad 2015$

Hurwitz monodromy and full number fields

DAVID P. ROBERTS and AKSHAY VENKATESH

The characteristic polynomial of the Adams operators on graded connected Hopf algebras

Marcelo Aguiar and Aaron Lauve

Secant spaces and syzygies of special line bundles on curves

MARIAN APRODU and EDOARDO SERNESI

Complex group algebras of the double covers of the symmetric and alternating groups

601

Christine Bessenrodt, Hung Ngoc Nguyen, Jørn B. Olsson and Hung P. TONG-VIET

Fano schemes of determinants and permanents

Melody Chan and NATHan Ilten

Triple intersection formulas for isotropic Grassmannians

VIJAY RAVIKUMAR

On the basepoint-free theorem for log canonical threefolds over the algebraic closure of a finite field

Diletta Martinelli, Yusuke NaKamura and Jakub Witaszek

The torsion group of endotrivial modules

JON F. CARLSON and JACQUES THÉVENAZ 